\title{
Les IGEM 2009
}

\section{La biologie synth-éthique}

\section{Sophia Häfner}

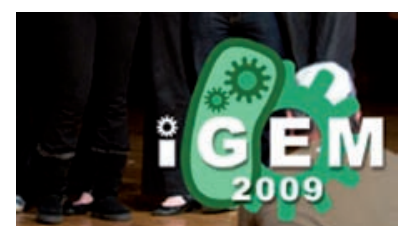

École normale supérieure et Médecine/Sciences, 45 , rue d'Ulm, 75005 Paris, France. sophia.hafner@ens.fr

\section{Les iGEM, une compétition de biologie synthétique ou life from scratch}

Il semblerait bien que nous assistions à la naissance d'une nouvelle tradition dans les événements scientifiques annuels: pour la troisième année consécutive, des équipes françaises de jeunes scientifiques ont participé à la compétition internationale de biologie synthétique iGEM (International Genetically Engineered Machine Competition), et Médecine/Sciences, comme les années précédentes ${ }^{1}$, dédie un article aux travaux extraordinaires des chercheurs de demain. Cette année se distingue par l'intérêt croissant des participants aux iGEM à l'aspect éthique de leurs travaux. Avec la publication récente de la création du premier organisme «artificiel » par le groupe de Craig Venter, la thématique se place parfaitement dans le contexte de l'actualité.

Les iGEM ont vu le jour en janvier 2003 au Massachusetts Institute of Technology (MIT) : une option d'enseignement proposait aux étudiants de développer des systèmes biologiques qui faisaient «clignoter» des cellules. Un an plus tard, le cours s'était transformé en une compétition, devenue internationale en 2005 et dont le rayonnement ne cesse de croître (13 équipes en 2005, 112 équipes et plus de 1700 participants venant du monde entier en 2009). Chaque année, des équipes d'étudiants prédoctorants issus de tous les domaines scientifiques et de formations diverses consacrent leur été à élaborer et concrétiser un projet qui consiste à créer des systèmes biologiques aux fonctions encore

${ }^{1}$ Häfner S. Compétition iGEM 2008 : deux équipes françaises! Med Sci (Paris) 2009; 25 : 524-8 et Bikard $D$, Képès $F$, au nom de l'équipe iGEM Paris. Succès de la première équipe française lors de la compétition iGEM de biologie synthétique. Med Sci (Paris) $2008 ; 24: 541-4$. inexistantes à partir de micro-organismes et de cellules dans lesquels sont assemblées des «bio-briques » fournies par le MIT. Drew Endy est l'un des créateurs du registre de ces composants biologiques standardisés (Registry of standard biological parts) du MIT : ce jeune «synthenthousiaste», comme l'appellent certains de ses détracteurs, passionné depuis l'enfance par les innombrables agencements des briques Lego, a conçu une impressionnante collection de plasmides, séquences codantes, promoteurs et amorces. Cette collection fonctionne sur le simple principe du «get some, give some » : n'importe qui a le droit de se servir dans cette collection à condition d'y inclure en retour les nouvelles constructions obtenues. C'est dans ce catalogue de «bio-briques» ou biobricks que puisent les étudiants du monde entier qui bricoleront à volonté de nouveaux organismes avant de s'affronter lors de la grande finale des iGEM au MIT, qui a lieu à l'automne à Cambridge.

\section{Le palmarès iGEM 2009}

Les projets des iGEM concernent en général les domaines de la santé ou de l'environnement: ainsi, en 2009, une équipe australienne a appris à des bactéries à reconnaître le mercure, des étudiants colombiens s'en sont servis pour dégrader des pesticides, et plusieurs équipes ont essayé d'envoyer des bactéries à la chasse aux cellules cancéreuses. L'équipe japonaise d'Osaka fait toutefois exception à la règle avec un projet plus artistique : leurs Salmonella s'assemblent en dessinant des mandalas ${ }^{2}$ dans des boîtes de Petri !

${ }^{2}$ Dessin ornementé circulaire à symétrie radiale. 
Le grand gagnant de l'année 2009 est l'équipe de Cambridge qui a conçu et caractérisé deux nouveaux outils pour fabriquer des biosenseurs précis, faciles à utiliser et, qui plus est, écologiques. Le projet $\varepsilon$. chromi consistait à transformer des souches de colibacilles en de petites machines à trois composants essentiels: un détecteur sous forme de promoteur sensible à un paramètre de l'environnement ( $\mathrm{pH}$, concentration, etc.), une construction capable d'ajuster la réponse au niveau de sensibilité désiré et une réponse sous forme de production d'un pigment ${ }^{3}$.

En deuxième position, l'équipe allemande de Heidelberg a su attirer l'attention de Drew Andy - ce n'est pas surprenant puisque les étudiants ont décidé d'ajouter à son catalogue de composants standardisés une rubrique «cellules de mammifères » en caractérisant les promoteurs connus et en développant un programme informatique capable de prédire l'efficacité d'un promoteur inconnu 4 .

\section{Cocorico : les équipes françaises}

\section{Le projet de l'équipe SupBiotech Paris: le système de double vectorisation}

SupBiotech ${ }^{5}$ propose une formation innovante en cinq ans qui permet à de jeunes bacheliers d'accéder à des postes de responsabilité dans toutes les fonctions des entreprises liées aux biotechnologies des secteurs de la santé, de l'innovation agroalimentaire ou encore de l'environnement. La participation d'une équipe SupBiotech aux iGEM 2009 a été à l'initiative d'Enguerrand Habran, étudiant en cinquième année. Passionné par les stratégies de vectorisation dans la lutte anti-cancer, il avait notamment travaillé à la réalisation d'une particule anti-cancer avec Patrick Couvreur, directeur de l'unité CNRS Physico-chimie pharmacotechnie biopharmacie, et lauréat du prix Galien Recherche 2009. Enguerrand s'est chargé de recruter une équipe de choc de onze étudiants (Figure l) qui se sont rapidement réparti les tâches en créant un groupe communication sous la responsabilité de Gaella Azzi, étudiante en cinquième année, spécialisée dans le marketing commercial en biotechnologie - chargé de la prospection des sponsors, des fournisseurs de matériel de laboratoire et également de l'organisation d'une conférence sur l'éthique. Chaque aspect du travail scientifique - cellule cible, phages et bactéries - était confié à un(e) ou plusieurs étudiant(e)s spécialisé(e)s en recherche

${ }^{3}$ http://2009.igem.org/Team:Cambridge

${ }^{4}$ http://2009.igem.org/Team:Heidelberg

${ }^{5}$ http://www.supbiotech.fr/ et développement tandis que Thimotée Khirkus ( $4^{\mathrm{e}}$ année de production en biotechnologie) était chargé de la partie modélisation. Le fruit d'une telle organisation, quasi professionnelle, est un projet non seulement intelligemment construit mais également bien présenté, comme le prouve le «wiki » (espace web des équipes au sein du site des iGEM) de SupBiotech en deux langues incluant une description détaillée de toutes les étapes du projet, dont une vidéo humoristique sur le quotidien du participant aux iGEM ${ }^{6}$.

Le projet est décrit dans l'Encadré 1 .

L'équipe Paris : quand les bactéries font des bulles...

Ce sont déjà les usual suspects français des iGEM: pour la troisième année, le Centre pour la recherche et l'interdisciplinarité (CRI), créé en 2005 comme un carrefour des sciences de la vie et des sciences exactes, cognitives et sociales, a engagé une équipe. Sous la tutelle experte d'Ariel Lindner, Guillaume Cambray et Samuel Bottani, treize étudiants d'horizons très divers (biologistes, mathématiciens, généticiens, sociologues, informaticiens et infectiologistes, issus de l'École normale supérieure, de l'École des Mines Paris Tech, de Supelec ou du master AIV ${ }^{7}$ ) se sont réunis fin avril 2009 pour un brainstorming savoureux que l'on peut découvrir sur le site officiel de l'équipe Paris ${ }^{8}$ : parmi les propositions, certaines ambitieuses (plasmides eucaryotes) et d'autres farfelues comme le bactowifi (des bactéries indiquant par leur fluorescence la force d'un signal électromagnétique) ou le bactocompass (des bactéries sensibles au champ magnétique, pour équiper les laboratoires de boussoles en boîtes de Petri...) a émergé message in a bubble, ou comment contrôler la communication bactérienne à longue distance grâce aux vésicules de la membrane externe.

Le projet est décrit dans l'Encadré 2.

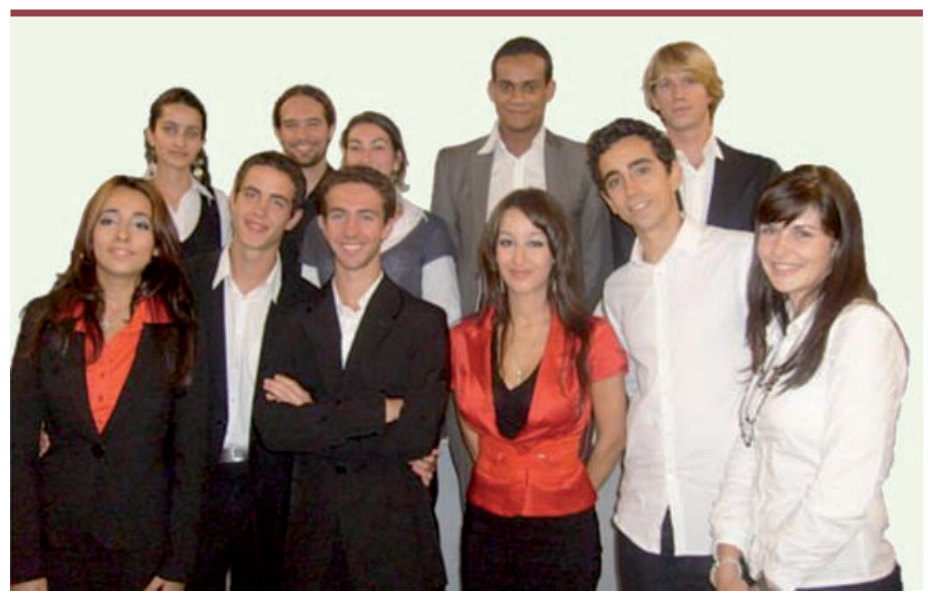

Figure 1. L'équipe SupBiotech. Premier rang, de gauche à droite : Ranya Jamali, Enguerrand Habran, Damien Parrello, Gaella Azzi, Cyprien Verseux et Emma Valette. Deuxième rang, de gauche à droite: Nassrine Lablack, David Charoy, Aurélie Négrel, Thierry Leclerc et Timothée Kirkhus.

${ }^{6}$ http://2009.igem.org/Team:SupBiotech-Paris

${ }^{7}$ Approches interdisciplinaires du vivant.

${ }^{8}$ http://2009.igem.org/Team:Paris 


\section{Le projet SupBiotech : thérapie phagique}

Ambitieux, les étudiants de l'équipe SupBiotech s'attaquent à la thérapie génique du cancer, avec pour objectif de corriger un des défauts génétiques associés, ici la perte par mutation de gènes suppresseurs de tumeurs. La solution élaborée par l'équipe SupBiotech consiste en un système de double vectorisation (DVS) (Figure 2): un premier vecteur bactérien atteint le tissu cible sans être éliminé par le système immunitaire et libère alors un second vecteur de type phagique contenant le plasmide thérapeutique qui sera internalisé par les cellules. Combattre le mal par le mal ? Le projet semble utopique à première vue, mais la description détaillée du scénario assez réaliste du groupe tend à convaincre du contraire. Le vecteur bactérien retenu était Mycobacterium avium, un homologue de Mycobacterium tuberculosis dont il ne partage pas la pathogénicité, puisqu'il n'entraîne que des symptômes cliniques légers, voire pas de symptômes chez le sujet sain immunocompétent. Le pathogène dispose de deux stratégies efficaces pour déjouer le système immunitaire de l'hôte: la composition de sa paroi cellulaire confère à M. avium une véritable armure, résistant à l'éthanol, aux détergents et aux antibiotiques classiques. De plus, ce micro-organisme intracellulaire facultatif peut infecter les macrophages et les cellules dendritiques responsables du déclenchement de la réponse immunitaire et la bloquer. Enfin, dernier atout, M. avium est un pathogène résident avec un tropisme préférentiel pour le foie, la rate et les poumons. Faible pathogénicité, résistance au système immunitaire et ciblage tissulaire font de M. avium un parfait candidat. quasi-intégralité de ses gènes via le gène $c l$ codant pour la protéine Repressor. En réponse à un stimulus, le phage peut réactiver son génome et entamer un cycle lytique aboutissant à la production de nouveaux phages. Pour le biologiste synthétique, cette alternance de phases lysogènes et lytiques s'appelle un interrupteur on/off et il est livré gratuitement avec le virus. Les systèmes permettant d'appuyer sur on au bon moment ne faisant pas défaut dans le répertoire des Biobricks, l'équipe SupBiotech a opté pour une induction à la doxycycline de la libération du phage selon un système tet/off classique, en associant les constructions de façon appropriée. Le bactériophage a été «bricolé » pour qu'il puisse reconnaître M. avium et non plus $\varepsilon$. coli et soit correctement endocytosé et ensuite relargué de l'endosome.

Dernière étape, l'encapsidation du plasmide thérapeutique dans le vecteur phagique lors de son assemblage est assurée en le dotant de séquences Cos. Les gènes phagiques ont été délestés de leurs extrémités Cos pour que seuls les gènes thérapeutiques soient transmis et pour priver le virus de toute capacité de réplication et de pathogénicité envers l'hôte commensal. L'importation nucléaire et la transcription de ces gènes sont à leur tour prises en charge par la séquence SV40 du Simian virus, comportant un signal de localisation nucléaire (NLS) et des sites de liaison à une poignée de facteurs transcriptionnels.

L'utilisation du vecteur viral résout le problème du passage des membranes en délivrant les gènes thérapeutiques directement dans la cellule d'intérêt. Le système d'induction à la doxycycline permet de contrôler à quel moment les gènes sont délivrés dans les cellules cibles, de préférence une fois que le vecteur tissulaire a atteint le site choisi. L’objectif précis de cette stratégie est de restaurer l'expression dans les cellules tumorales d'un certain nombre de gènes suppresseurs de tumeurs ( $p 53, R B 1, P T \varepsilon N$, etc.) dont la mutation prive la cellule d'une réponse apoptotique salutaire, la rendant ainsi immortelle. Puisqu'ils se trouvent sous le contrôle de leur promoteur naturel, ces gènes ne seront pas activés dans les cellules saines.

L'équipe SupBiotech a bien pris soin de démontrer que le projet est à la fois réaliste et réalisable. Les expériences préliminaires de l'équipe montrent que la création du phage recombiné et l'infection de cellules eucaryotes sont possi-

Figure 2.

Une fois le tissu cible atteint, M. avium doit passer le relais au second vecteur, porteur des gènes thérapeutiques et capable de pénétrer dans les cellules (Figure 2). C'est le bactériophage $\lambda$ qui a été choisi, un virus à $A D N$ procaryote qui s'attaque habituellement à $\varepsilon$. coli. Une fois entré dans la bactérie, le phage entre en phase lysogène, intègre son unique chromosome circulaire dans l'ADN de l'hôte et désactive la bles et que l'insertion du gène $p 53$ induit l'apoptose dans des lignées cancéreuses en culture. Pour couronner le tout, une modélisation mathématique et un simulateur permettent de suivre l'évolution de la tumeur après ce transfert DVS et de calculer à quel moment la doxycycline doit être injectée pour éliminer la tumeur dans son intégralité. 


\section{Quand l'éthique s'en mêle}

JCVI-syn1.0...

«Nous rapportons la conception, la synthèse et l'assemblage du JCVI-syn 1.0... $\gg$ ScienceExpress du 20 mai 2010. Quinze ans, 40 millions de dollars et 20 personnes : c'est le prix d'un rêve. Celui de J. Craig Venter, pour être précis. Bien connu des médias pour avoir séquencé le génome humain en 2001 , un certain manque de modestie et la volonté d'être le premier à créer la vie artificielle dans une boîte de Petri. Moins connu pour avoir séquencé en 1995 le plus petit génome, le chromosome de 600000 paires de bases de Mycoplasma genitalium. Célèbre maintenant pour avoir donné vie à une bactérie avec un génome entièrement synthétique ${ }^{9}$, dans lequel les auteurs ont laissé des marques - des noms, des adresses mail et même une citation de James Joyce encodés en ATCG - du coup on pourra lire «to live, to err, to fall, to triumph, to recreate life out of life ».

Lors des premières compétitions iGEM, une seule équipe, celle du MIT, avait publié en 2005 un court texte sur la sécurité biologique (biosafety). En 2008, l'iGદM crée un prix récompensant les «meilleurs progrès en pratiques humaines »; il fut décerné à l'équipe de Heidelberg, sélectionnée parmi 14 équipes qui avaient traité cet aspect éthique. Cette année, plusieurs équipes ont dédié une partie de leur temps et de leur travail écrit aux questions éthiques. Sara Aguiton, notamment, qui a travaillé avec l'équipe Paris, a été distinguée par un Prix spécial du jury pour son approche des questions éthiques et sociales.

L'origine du lien entre éthique et biologie synthétique est ancienne: créer la vie a été le rêve et le cauchemar des hommes depuis des siècles et a inspiré des expériences curieuses comme le galvanisme d'Alessandro Volta en $1780^{10}$ ou le Frankenstein de Mary Shelley (1818). Et même le journal scientifique prestigieux Nature fait remarquer que, si bien des techniques utilisées par la science ont été considérées comme un affront envers Dieu, celle qui se prête le plus à ce reproche est la biologie synthétique. La société redoute plus I'utilisation des nouvelles technologies par des bioterroristes que la destruction du monde par une créature créée dans une boîte de Petri (même s'il s'agit d'une vision amplement exploitée par les romanciers et l'industrie cinématographique). En effet, un journaliste du Guardian (journal anglais) avait commandé une partie du génome du virus de la variole auprès d'une des firmes qui synthétisent l'ADN sur commande et l'avait reçu sans problème. Depuis, l'industrie des gènes synthétiques s'est certes engagée à comparer toute séquence

\footnotetext{
${ }^{9}$ Legrain P. La vie créée au laboratoire? Amis biologistes, encore un petit effort! Med Sci (Paris) 2010 ; $26: 559-60$.

${ }^{10}$ En 1800, Alessandro Volta inventa la première pile électrique. Par une lettre du 2 juillet 1801, il fit connaître à Dieudonné Dolomieu ses résultats les plus récents (voir le manuscrit comprenant la description et les dessins de la pile de poche et sa traduction). C'est alors que le premier consul, Bonaparte, l'invita à présenter son invention à Paris. Le 16 brumaire an X (7 novembre 1801), la première classe de I'Institut national des sciences et des arts entendit la lecture faite par Volta sur la théorie du galvanisme et particulièrement sur la nature du fluide galvanique. À l'issue de cette séance, Bonaparte proposa que l'assemblée donnât une médaille d'or au citoyen Volta, ce qui fut réalisé à la suite d'un rapport de JeanBaptiste Biot, lu le 11 frimaire an X (2 décembre 1801) (site de l'Académie des sciences).
}

commandée avec la liste intégrale des pathogènes connus à l'heure actuelle, mais le malaise persiste.

\section{iGEM s'intéresse à l'éthique}

L'équipe de Valence (Espagne) n'a pas seulement fabriqué un écran biologique - dont chaque pixel est une levure capable de répondre à un stimulus électrique par l'émission de lumière ${ }^{11}$ - mais elle a aussi, à l'occasion des iGEM, réuni dans un ouvrage intitulé Sins, ethics and biology, a comprehensive approach - téléchargeable sur leur site - la totalité des projets antérieurs des iGEM, des entretiens avec des experts du domaine (Marcus Schmidt et Antoine Danchin ${ }^{12}$ ) et un sondage à grande échelle auprès des autres équipes ${ }^{13}$. La présentation, d'une qualité remarquable, comporte la synthèse d'une cinquantaine d'articles et de nouvelles traitant de la biologie synthétique et de ses implications éthiques publiés dans des journaux spécialisés, et des travaux effectués par les participants des iGEM entre 2005 et 2008. D'autre part, l'équipe a effectué un sondage (1 288 participants, le plus important à ce jour) sur la biologie synthétique, confrontant d'une part les personnes «informées», en l'occurrence les participants des iGEM 2009, et d'autres «non informées » parmi les scientifiques ou non-scientifiques, qui ont été contactés via des sites internet connus ou des réseaux sociaux, notamment Facebook. Leur questionnaire va de la définition de la biologie synthétique aux questions critiques et fondamentales, par exemple : «En supposant que l'homme soit capable de créer une vie artificielle, en avons-nous le droit? » et «À quel point la biologie synthétique est-elle dangereuse ou bénéfique?», sans omettre la question de la brevetabilité des outils génétiques. Les résultats, longuement discutés dans le travail, nous révèlent quelques aspects de la vision actuelle des ingénieurs du vivant. La question du droit à la création de la vie est notamment controversée : $63 \%$ des participants au sondage estiment que oui, I'homme peut produire des créatures artificielles ( $66 \%$ des informés, $57 \%$ des non-informés), $26 \%$ que non et $11 \%$ s'estiment incapables de répondre à la question compte tenu d'implications morales et religieuses. Les étudiants de Valence concluent que la définition exacte de la biologie synthétique est encore mal connue par les non-scientifiques et que les opinions concernant la propriété intellectuelle et la création de la vie reposent sur des convictions acquises très tôt et peu modifiables. Ils

\footnotetext{
${ }^{11}$ http://2009.igem.org/Team:Valencio

12 Danchin A. Saurons-nous contruire une bactérie? Med Sci (Paris) 2008; $24: 533-40$.

${ }^{13}$ http://2009.igem.org/Team:Valencia/Human
} 


\section{L'équipe Paris : quand les bactéries font des bulles...}

Faire main basse sur le système de communication bactérien

À l'image des cellules d'un organisme multicellulaire, les bactéries coordonnent leurs activités (croissance, division) en communiquant les unes avec les autres au sein de la population globale. Ce quorum sensing fait intervenir de petites molécules chimiques, les acylhomosérines lactones ( $\mathrm{AHL}$ ), dont le seul défaut réside dans leur faible solubilité dans l'eau, qui restreint la portée du signal dans un environnement aqueux. Quand il s'agit en revanche d'envoyer des toxines dans des cellules hôtes, les bactéries Gram négatives ne manquent pas d'imagination. Elles sont également capables de former des vésicules à partir de leur membrane extérieure dont le contenu périplasmique peut comporter des facteurs de virulence, des immunomodulateurs et des toxines. Ces vésicules, capables de franchir de longues distances pour atteindre leur cible de manière concentrée et spécifique, jouent aussi un rôle prépondérant dans l'acquisition de nutriments, la formation de biofilms et la réponse au stress des bactéries. Les bases d'un système de communication efficace étant naturellement présentes, il suffisait de faire main basse sur le système...

\section{Cacheter la lettre et la donner au facteur}

L'objectif du projet est d'envoyer un message à une cellule cible via la machinerie des vésicules de la membrane externe. Exemple choisi par l'équipe : faire parvenir la protéine FecA (transporteur de fer) à une population FecA-mutante, dans laquelle le FecA reçu par courrier de vésicules activerait l'opéron FecABCD dans lequel une protéine étiquette fluorescente a été introduite. Diverses étapes ont dû être franchies (Figure 3): (1) insérer le message protéique dans le périplasme ou la membrane externe pour assurer son incorporation dans les vésicules; l'équipe a opté pour une stratégie classique : la cytolysine a (ClyA) - une toxine à formation de pores capable de s'intégrer dans la membrane de la cellule hôte et la rendant perméable à l'injection d'autres toxines - ClyA est dotée d'un peptide signal permettant sa translocation du cytoplasme vers la membrane externe par la voie Tat (twin-arginine translocation) et est enrichie dans les membranes des vésicules produites. En se basant sur des études prouvant que la fusion de ClyA avec des protéines rapporteurs n'entrave pas la translocation, les Parisiens fusionnent «leur» ClyA avec la RFP (red fluorescent protein) ou FecA et placent la construction sous le contrôle activateur de l'arabinnose et inhibiteur du glucose. (2) Faire faire des bulles à la bactérie, ce qui fut fait en provoquant la déstabilisation de la membrane via le système Tol/Pal, impliqué dans l'ancrage de la membrane externe à la membrane interne et à la couche de peptidoglycanes ${ }^{1}$. Encore fallait-il doser la production de vésicules de façon à éviter qu'elle ne soit excessive et létale pour la bactérie, et ne la déclencher qu'une fois la protéine signal bien en place dans la membrane externe, ce qui a été résolu en introduisant un système «retard » dans les plasmides $^{2}$ (Figure 3).

\section{Le destinataire du message}

Dernière étape, il fallait concevoir un système d'adressage et de réception des vésicules par la population bactérienne receveuse, en l'occurrence la population FecA-. L'équipe parisienne propose deux variantes: le dimère Jun/Fos ou la protéine virale G3P. Jun et Fos font partie du complexe protéique AP-1, un facteur de transcription eucaryote. Grâce à des domaines leucine-zipper, la formation d'homodimères Jun/Jun ou d'hétérodimères Jun/Fos est possible et deux mutations dans le domaine leucine-zipper suffisent pour empêcher la formation des homodimères. Dans le modèle élaboré, les cellules donneuses expriment Jun et les cellules receveuses Fos. Puisqu'il s'agit de protéines normalement nucléaires, c'est au tour de l'autotransporteur AIDA (adhesin involved in diffuse adherence) de les amener cette fois-ci à la surface de la membrane externe sous forme de protéines de fusion. La protéine virale G3P est exposée à la surface des bactériophages filamenteux (par exemple M13) et leur permet de fusionner de manière efficace avec la paroi bactérienne. Le processus de fusion ayant lieu au niveau des pili, la population donneuse serait dépourvue de pilus au contraire de la population receveuse. Ne laissant rien au hasard, l'intégrité des processus, notamment la réception du signal, a été vérifiée par simulation informatique, démontrant la solidité du mécanisme proposé. Les efforts en valaient visiblement la peine puisque le jury des iGદM a décerné une médaille d'or au projet scientifique.

${ }^{1}$ Henry T, Pommier S, Journet L, et al. Improved methods for producing outer membrane vesicles in Gram-negative bacteria. Res Microbiol 2004 ; 155 : 437-46.

${ }^{2}$ Hooshangi S, Thiberge S, Weiss R. Ultrasensitivity and noise propagation in a synthetic transcriptional cascade. Proc Natl Acad Sci USA 2005 ; 102 : 3581-6.

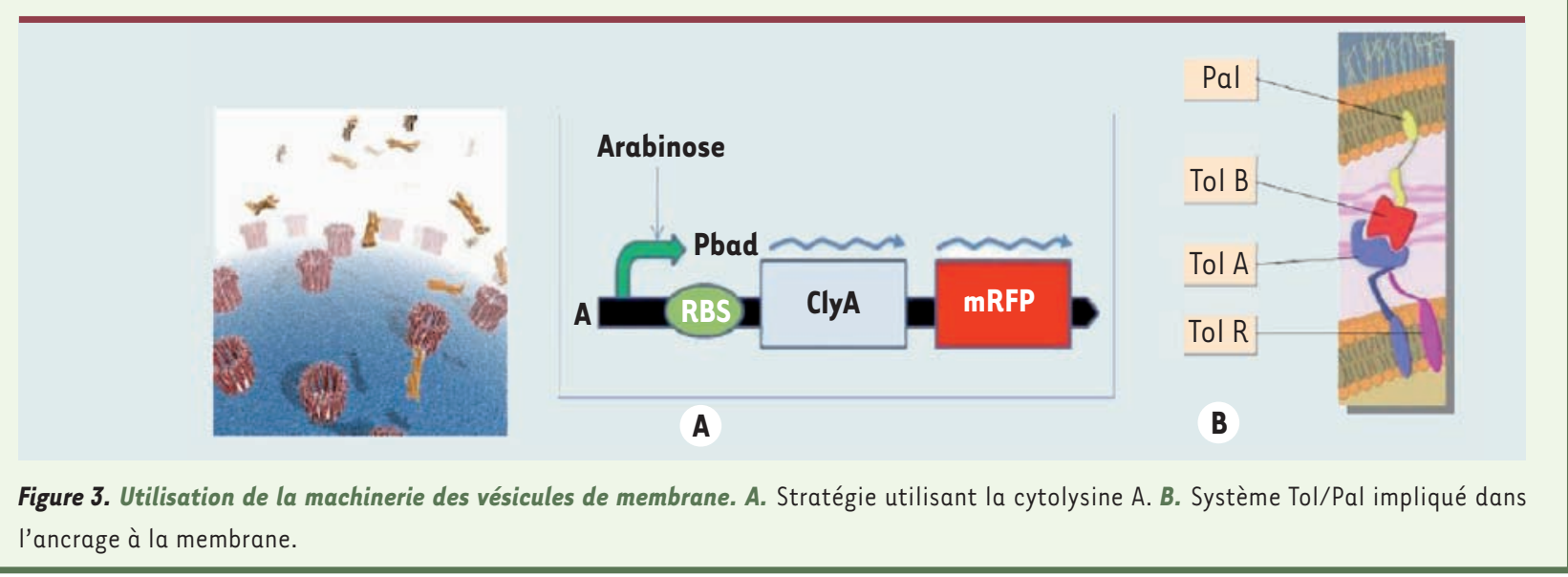


prônent en conséquence une meilleure dissémination de l'information dans ce domaine auprès du grand public. Mais revenons à nos deux équipes françaises qui ne sont pas non plus passées à côté de la nouvelle tendance, bien au contraire.

\section{SupBiotech-Paris et le débat éthique sur les enjeux de la biologie synthétique}

L'équipe SupBiotech-Paris a organisé à cette occasion un débat éthique intitulé «Les enjeux de la biologie synthétique, état des lieux, état d'esprit » le 9 octobre 2009, motivée par deux constats: "l'émergence et le développement rapide de [la biologie synthétique] nécessitent une réflexion, afin de mettre en place un système de réglementation prêt dans les 5 à 10 ans pour des pratiques sûres » et «c'est la première fois que l'homme est confronté à la possibilité de créer de nouvelles formes de vie » (François Le Fèvre). Parmi les invités figuraient des noms célèbres tels que Bernard Baertschi, maître d'enseignement et de recherche à l'Institut d'éthique biomédicale et au département de philosophie de l'Université de Genève, Willy Rozenbaum, le codécouvreur du virus du VIH (virus de l'immunodéficience humaine), Thierry Magnin, docteur en théologie et professeur de physique de l'École des Mines de Saint-Étienne, Lluis M. Mir, directeur de recherche au CNRS et François Le Fèvre, chercheur au Commissariat à l'énergie atomique (CEA). Les vidéos et une synthèse soigneuse du débat peuvent être consultées sur le site de l'équipe ${ }^{14}$. Les thématiques abordées peuvent être classées en deux grandes catégories: les réflexions d'ordre métaphysique et les problèmes pratiques liés à l'application de la biologie synthétique. En tête dans la première figure la question de la finalité de la biologie synthétique : un désir d'amélioration et de perfection du vivant? «Comment pourrait-on s'en passer si on veut aller vers une amélioration de la condition humaine?» demande Willy Rozenbaum. Suit le problème de la représentation du vivant que Thierry Magnin exprime à merveille avec la phrase «Comment puis-je reconnaître une certaine dignité du Vivant si tout est fabriqué par blocs ? ». Il est certain que la science procède depuis toujours et sans scrupules à une démystification de la vie, depuis qu'en 1809 Lamarck a mis fin au théorème du vitalisme de Platon et Aristote en affirmant dans sa Philosophie zoologique que la vie, aussi compliquée soit-elle, était un phénomène purement physique. Pour Thierry Magnin, le risque réside dans l'aspect quelque peu ludique de la biologie synthétique (avec une pensée pour les briques Lego de Drew Endy): «Ce avec quoi

\footnotetext{
${ }^{14}$ http://2009.igem.org/Team:SupBiotech-Paris/Biologie_Synthetique\#drapeau
}

j'ai l'habitude de jouer, j'ai souvent un peu de mal à le respecter ». L'homme est naturellement curieux, saura-t-il vraiment s'imposer des limites?

Côté pratique, les étudiants ont surtout planché sur la question de la propriété intellectuelle et du dilemme entre la menace de bioterrorisme que pourrait faciliter un libre accès au savoir et le risque de ralentir l'innovation en permettant de breveter les «briques» biologiques, voire les organismes créés synthétiquement. Cela dit, la majorité des élèves de SupBiotech interrogés par leur équipe iGEM s'est prononcée en faveur des brevets. Quant à la question de savoir si nous serons en mesure de contrôler les systèmes créés, les avis divergent. La moitié des élèves croient l'homme capable de contrôler le vivant et $32 \%$ estiment que nous ne maîtriserons pas la propagation des organismes créés.

\section{L'équipe Paris : analyse éthique}

\section{et sociale de la biologie synthétique}

Dans le cas de l'équipe Paris, l'initiative venait de l'extérieur. Plus précisément, elle venait d'une jeune étudiante de l'École des hautes études en sciences sociales (EHESS). Sara Aguiton, âgée de 23 ans, est étudiante en master 2 de recherche au Centre Alexandre Koyré en sociologie et histoire des sciences et des techniques, sous la direction de Dominique Pestre. Titulaire d'une licence de philosophie acquise à la Sorbonne, elle consacre son travail de mémoire à l'étude anthropologique et sociologique de la biologie synthétique. Les iGEM 2009 représentaient donc un sujet d'étude idéal pour un stage facultatif, avec une quinzaine d'étudiants comme cobayes pour répondre à la question: comment se construit un projet scientifique de nos jours? Sara avait contacté Ariel Lindner, encadrant de l'équipe Paris, au tout début de la session 2009, et elle assista à la naissance du projet et à sa mise en œuvre sur les ordinateurs et à la paillasse. Mais son travail était loin d'être contemplatif; elle avait préparé tout un arsenal d'entretiens individuels, de présentations et de réunions pour l'équipe. Les thématiques abordées et le résultat des discussions ont été soigneusement détaillés dans un «cahier de manip éthique ${ }^{15}$ » et centrés autour de la question de la nécessité d'une approche éthique des sciences et en particulier de la biologie synthétique. En parallèle, Sara s'était chargée de la rédaction d'un rapport d'une cinquantaine de pages intitulé An ethical and sociological analysis on synthetic biology.

Ce travail remarquable a été estimé à sa juste valeur par le jury des iGEM 2009 qui lui a décerné le Prix spécial du jury pour les approches éthiques et sociales, événement qui a fait la une du journal Le Monde en novembre $2009^{16}$. Curieusement, la collaboration avec les étudiants n'était pas évidente comme nous l'a confié Sara lors de notre rencontre. Non qu'ils aient été réfractaires aux idées, mais les réactions restaient passives. II était difficile de les motiver pour la discussion et la constitution du blog éthique, et leur commentaire sur la page de présentation des participants se résumait à «utilise des mots éthiques simples quand tu parles à un groupe de scientifiques, s'il te

\footnotetext{
15 http : \\2009.igem.org/Team:Paris/Ethics_ethicalbook"top

16 «Un gène éthique qui vaut de l'or», par Annie Kahn, Le Monde du 28 novembre 2009.
} 
plaît ». Cette remarque met le doigt sur la plaie. En effet, les notions d'éthique dont dispose l'étudiant scientifique sont plus que vagues, faute d'enseignement dédié. La seule formation non scientifique solidement incorporée dans de nombreux parcours scientifiques est le management et la gestion d'entreprise. L'éthique et la philosophie des sciences sont, et encore quand elles existent, des options facultatives. Et supprimer les cours d'histoire-géographie en terminale scientifique n'arrangera certainement pas les choses... La tendance est de former des machines intellectuelles dépourvues de toute capacité de communication, réflexivité et attitude critique.

Pourtant, un étudiant de l'ćcole des Mines a eu une illumination soudaine lors d'une discussion, raconte Sara: auparavant il ne s'était guère rendu compte qu'un nombre non négligeable d'exercices de cours prenaient comme exemple la chute de bombes et les trajec- toires de missiles. Anecdote triste mais vraie qui pose la question fondamentale de la responsabilité de la science mais qui démontre aussi à quel point l'abstraction mathématique constitue un filtre devant les problèmes. $\diamond$

IGEM 2009: synthetic biology and ethics

\section{CONFLIT D'INTÉRÊTS}

L'auteur déclare n'avoir aucun conflit d'intérêts concernant les données publiées dans cet article.
TIRÉS À PART

S. Häfner

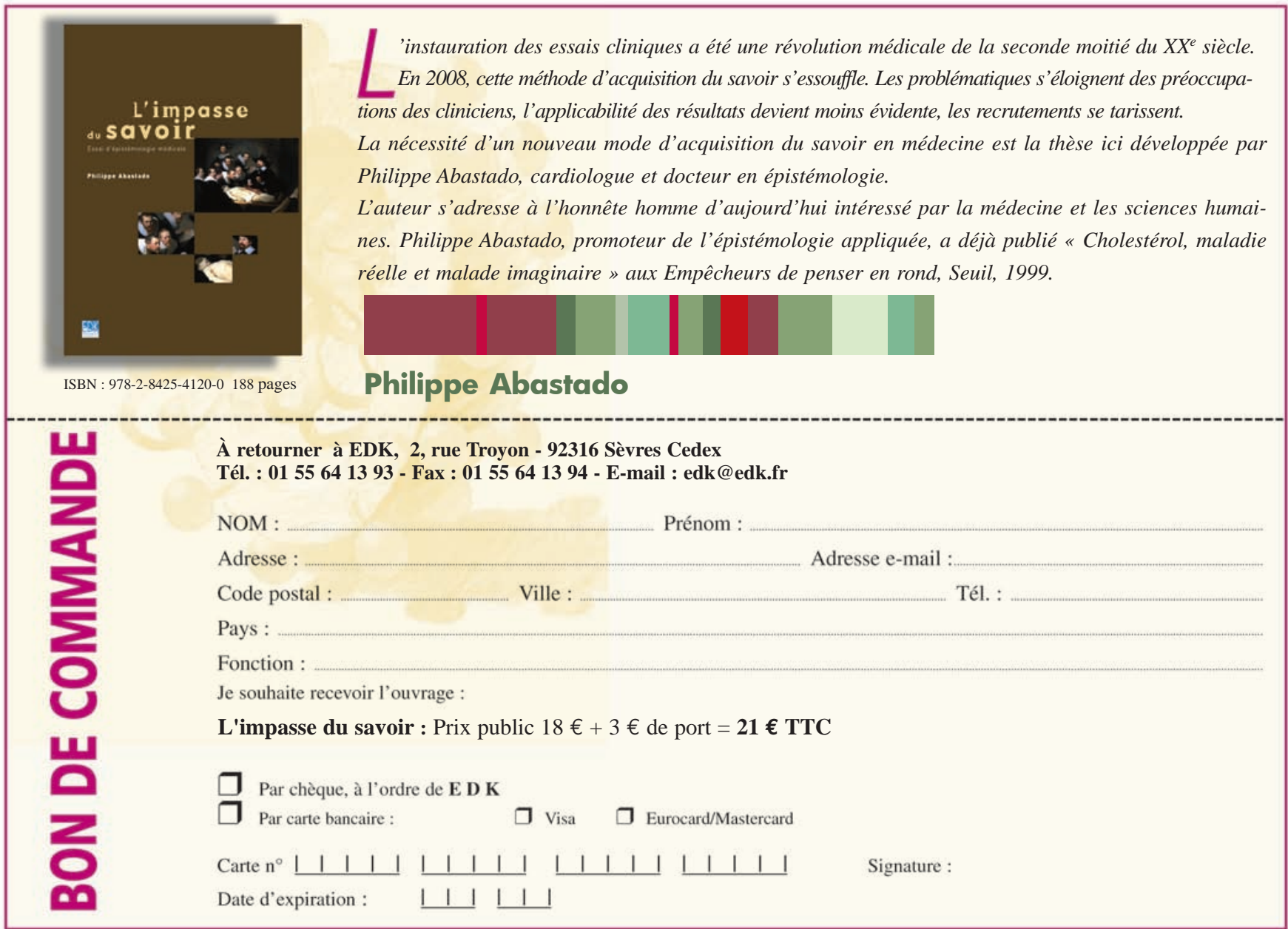

